\title{
Recycling of Industrial Metals and Materials: Trends in Recent Research-Part I
}

\author{
DIRK E. VERHULST (i), ${ }^{1,4}$ FISEHA TESFAYE, ${ }^{2,5}$ \\ and MARK E. SCHLESINGER ${ }^{3,6}$
}

\begin{abstract}
1.-Reno, NV, USA. 2.-Johan Gadolin Process Chemistry Centre, Åbo Akademi University, Piispankatu 8, 20500 Turku, Finland. 3.-Department of Materials Science and Engineering, Missouri University of Science and Technology, 1400 N. Bishop Ave., Rolla, MO 65401, USA. 4.—e-mail: dirkverhulst@gmail.com. 5.—e-mail: fiseha.tesfaye@abo.fi. 6.—e-mail: mes@mst.edu
\end{abstract}

Environmental concerns and the need for energy savings are presently the most important drivers of research in extractive metallurgy. Recycling fits into this general picture: not only the re-processing of post-consumer products, but also various improvements in a flowsheet that send intermediate products and residues back as feedstock to a process. The present series of papers is mostly devoted to the second aspect.

The research presented here, as most research in this field, is not groundbreaking or disruptive. Instead, it consists of incremental steps that have already been considered in the past but make practical sense and have now become very important because of changes in economics and regulations. We trust the papers give up-to-date information as well as an indication of general trends.

Because of the abundance of interest in the topic, publication has been split over two issues of JOM. A first series of papers appears as Part I in the present issue. Another series will be published as Part II in a few months.

The seven papers presented here cover hydrometallurgy, pyrometallurgy and mineral processing. The first four papers all propose optimized pyrometallurgical processing steps that will increase the recovery of metals in the subsequent hydrometallurgical stage.

"Flotation of a Low-Grade Zinc Oxide Ore After Surface Modification at High Temperature" by L.-Z. Chen et al. shows how sulfidation at higher temperature improves flotation characteristics, even in the presence of a high percentage of slimes.

Dirk E. Verhulst, Fiseha Tesfaye, and Mark E. Schlesinger are the JOM advisors for the Recycling and Environmental Technologies Committee of the TMS Extraction \& Processing and the Light Metals Divisions, and guest editors for the topic Recycling of Industrial Metals and Materials: Trends in Recent Research in this issue.
The next paper "Recycling Utilization of ZincBearing Metallurgical Dust by Reductive Sintering: Reaction Behavior of Zinc Oxide" by Wei Lv et al. discusses improvements in the recycling of zinc residues, in this case blast furnace dust, using a pyrometallurgical process with well-controlled conditions of oxidation reduction.

The third paper also addresses roasting as a pretreatment step. The title is "Sodium Salt-Assisted Reductive Roasting for Separation and Enrichment of Valuable Components from Lateritic Iron Ore" by Mingxia Liu et al. The study gives new information on the possibility of using sodium salts to extract a high-chromium phase and directlyreduced iron from laterite residues.

The article "Effect of Roasting Characteristics of Vanadium-Rich Slag on Its Vanadium Leaching Behavior" by Wei Li et al. investigates the effect of roasting conditions on the subsequent leaching of vanadium from vanadium-rich slags. The authors have performed thermodynamic calculations using FactSage to determine the formation of oxides in the selected roasting conditions, which they correlated with the leaching experiments. Such calculations give more information than a set of stoichiometric equations and should be considered in all discussions of complex chemical equilibria.

The next paper "Titanium Recovery from TiBearing Blast Furnace Slag by Alkali Roasting and Acidolysis" by Siqi He et al. also describes the recovery of metal from a slag phase. The key challenge is doing this in a way that is economically competitive with the recovery of titanium from primary ores.

In a somewhat different field is "Recovery of Apatite from Ore Slimes Using Centrifugal Heavy Media Separation" by Mahmut Altiner. The material recycled is of relatively low value, and environmental concerns may be the main driver. All the 
three factors studied had an important effect on the grade and the recovery of the apatite product. The application of a Box-Behnken design made it possible to include non-linear interactions between factors without requiring too many experiments.

The last paper is titled "Review of the Effect of Oxygen on Titanium and Deoxygenation Technologies for Recycling of Titanium Metal" by Yang Xia et al. Titanium scrap is difficult to remelt without absorbing oxygen, and removing this oxygen is a challenge. Several proposed and commercial technologies for oxygen removal are described.

The following list summarizes the papers being published under the topic of "Recycling Methods for Industrial Metals and Minerals: Part I". To view or download any of the papers, follow the URL http:// link.springer.com/journal/11837/71/9/page/1 to the table of contents page for the September 2019 issue (vol. 71, no. 9).

- "Flotation of a Low-Grade Zinc Oxide Ore After Surface Modification at High Temperature" by Lu-Zheng Chen, Cong-bing Wang, Yong-xing Zheng, Jin-fang Lv, Zhen-ning Lai, and Jie Pang
- "Recycling Utilization of Zinc-Bearing Metallurgical Dust by Reductive Sintering: Reaction Behavior of Zinc Oxide" by Wei Lv, Min Gan, Xiaohui Fan, Zhiyun Ji, Xuling Chen, Jiawen Yao, and Tao Jiang

- "Sodium Salt-Assisted Reductive Roasting for Separation and Enrichment of Valuable Components from Lateritic Iron Ore" by Mingxia Liu, Changgen Wang, Jun Luo, Mingjun Rao, Guanghui Li, and Tao Jiang

- "Effect of Roasting Characteristics of VanadiumRich Slag on Its Vanadium Leaching Behavior" by Wei Li, Haiyan Zheng, and Fengman Shen

- "Titanium Recovery from Ti-Bearing Blast Furnace Slag by Alkali Roasting and Acidolysis" by Siqi He, Tongjiang Peng, and Hongjuan Sun

- "Recovery of Apatite from Ore Slimes Using Centrifugal Heavy Media Separation" by Mahmut Altiner

- "Review of the Effect of Oxygen on Titanium and Deoxygenation Technologies for Recycling of Titanium Metal" by Yang Xia, Jinlong Zhao, Qinghua Tian, and Xueyi Guo. 